\title{
Guidelines for quality control of PET/CT scans in a multicenter clinical study
}

\author{
Ivalina Hristova ${ }^{1,4^{*}}$, Ronald Boellaard ${ }^{2,4}$, Paul Galette ${ }^{5}$, Lalitha K. Shankar ${ }^{6}$, Yan Liu ${ }^{4,7}$, Sigrid Stroobants ${ }^{4,8}$, \\ Otto S. Hoekstra ${ }^{4,9}$ and Wim J.G. Oyen ${ }^{1,3,4}$
}

* Correspondence:

ivalinahr@gmail.com

${ }^{1}$ Department of Nuclear Medicine, Radboud University Medical Centre, Geert Grooteplein-Zuid 10, 6525 GA Nijmegen, The Netherlands ${ }^{4}$ European Organization for Research and Treatment of Cancer, Imaging Group, Brussels, Belgium Full list of author information is available at the end of the article

\begin{abstract}
To date, there is no published detailed checklist with parameters referencing the DICOM tag information with respect to the quality control (QC) of PET/CT scans. The aims of these guidelines are to provide the know-how for effectively controlling the quality of PET/CT scans in multicenter studies, to standardize the QC, to give sponsors and regulatory agencies a basis for justification of the data quality when using standardized uptake values as an imaging biomarker, to document the compliance with the imaging guidelines, to verify the per protocol population versus intent to treat population, and to safeguard the validity of multicenter study conclusions employing standardized uptake value (SUV) as an imaging biomarker which is paramount to the scientific community. Following the proposed guidelines will ensure standardized prospective imaging QC of scans applicable to most studies where SUVs are used as an imaging biomarker. The multitude of factors affecting SUV measurements when not controlled inflicts noise on the data. Decisions on patient management with substantial noise would be devastating to patients, ultimately undermine treatment outcome, and invalidate the utility of SUV as an imaging biomarker usefulness. Strict control of the data quality used for the validation of SUV as an imaging biomarker would ensure trust and reliability of the data.
\end{abstract}

\section{Background}

The variability and reproducibility of standardized uptake value (SUV) measurements and the factors affecting SUV as an imaging biomarker (IB) have been well studied and documented [1-6]. Major work has been done in the area of proposing criteria and methodology for the SUV measurements in multicenter studies [7-14]. However, limited information is available on the methodology and strategy used to verify the basics behind the factors affecting SUVs, and to date, no guidelines have been proposed offering detailed step-by-step quality control (QC) for positron emission tomography (PET) scans, with the goal to standardize reporting of imaging guidelines (IG) and protocol compliance. The aims of these guidelines are to systematize the reporting of QC in multicenter PET studies, in order to properly document it in all clinical trials involving SUVs as an IB used for the study end points, to provide investigators with the know-how for handling IG deviation related queries, and to provide the sponsors with a method to determine the size of the per protocol population (PPP) cohort. This will ensure

(c) The Author(s). 2017 Open Access This article is distributed under the terms of the Creative Commons Attribution 4.0 International License (http://creativecommons.org/licenses/by/4.0/), which permits unrestricted use, distribution, and reproduction in any medium, provided you give appropriate credit to the original author(s) and the source, provide a link to the Creative Commons license, and indicate if changes were made. 
standardized prospective imaging QC and review of scans which could be applied in most studies where SUVs are used as an IB. These guidelines are indicative in nature and may be subject to some variations depending on the complexity of the imaging indication, pharmacokinetics, and pharmacodynamics of the studied compound, the treatment technique, the number of patients enrolled in each trial, and the sponsor's commitment.

\section{Background}

\section{Key players and components}

The majority of industry-sponsored clinical study implementation starts with an investigator meeting, which usually includes the patient-recruiting physician and the study coordinator at the investigating center. The aim of the imaging manual is to provide a detailed educational session for those two key players on the study protocol and the required documentation. While usually multiple departments are involved in the patient management in the clinical study (i.e., pathology, radiology, and laboratory), it is expected that the recruiting physician and the study coordinator would pass on to the rest of the departments at his/her center all relevant information, a copy of the protocol, and any specific guidelines, instructions, forms, and techniques to be used in the patient management or patient data management, including biological specimens, images, and so on. The recruiting physician is responsible to ensure that all involved parties have training on the protocol and are qualified to care for the patients in the study and manage the data.

With the increasing number of clinical studies designed to use surrogate end points such as progression free survival (PFS), more and more studies use imaging biomarkers. Imaging biomarkers can basically be divided into morphological and functional qualitative and quantitative readouts. The PET-based SUVs are semiquantitative measurements based on functional imaging often used with a different purpose in a clinical study: patient inclusion/exclusion, response monitoring, follow-up, and as prognostic and predictive IB. In light of the increased use of well established (e.g., fluorodeoxyglucose (FDG)) and more recent PET radiopharmaceuticals (e.g., fluorothymidine) included in clinical trials, it should be clearly outlined to the PET imaging department that scan parameters will be checked during the QC of the scans by the sponsor or its designee. These details are usually not discussed in the study protocol and are not clear to the sites. Yet, often, it is the study coordinator who is tasked to reply to imaging-related queries. PET-related queries could be very technical with respect to artifacts, scan acquisition, and reconstruction parameters or could be patient- and visit-oriented. For the latter, the study coordinator should be able to provide the information without the assistance of the PET department. However, in cases where the queries quote specific scan technical parameters, usually the PET department would be the most appropriate to reply. This implies that the PET personnel at the site should be well acquainted with the imaging guidelines and the protocol end points, in order to minimize the number of queries for deviations. Furthermore, the PET personnel should be aware of the factors affecting SUVs and the implication of not following the imaging guidelines on the end points of the study. Not following the imaging guidelines could lead to a study failure [6]. 
Figure 1 outlines the proposed steps to ensure data integrity for studies where SUVs are used as an IB in the clinical trial end point(s).

\section{Prior to the selection of sites}

Imaging Facility Questionnaire (IFQ) is a questionnaire documenting the infrastructure, personnel, and technical facilities available at the site's PET imaging department. It allows for a verification that a site meets the minimum requirements imposed by the sponsor and that it is equipped for delivering the required quality of the scans. The IFQ can be used for selecting sites for participation in a study involving specific imaging techniques and modalities.

Dummy run or dry run (DR) are scans sometimes required to be performed either on a subject or a phantom prior to enrolling patients to participate in a clinical study. If a subject scan is submitted for this purpose, a proper consent should be in place. DR is a "clinical mock test" undertaken at each site before the site is authorized to enroll patients, or at a very early phase of the study, depending on the sponsor's decision and the study design. This mock test scan is reviewed by the sponsor or its designee, who would verify scanner performance, image quality, and consistency of acquisition/reconstruction parameters with the imaging guidelines and study protocol. The DR is usually not analyzed further. The DR may be specific to a study allowing up-front checking of the site's ability to comply with the protocol and the IG. The DR could also identify the presence of possible ambiguities in the protocol and the IG and assist in the implementation of corrective measures to guarantee a satisfactory selection, treatment, and followup in study patients. DR submission also checks to ensure that scan upload is possible via electronic transfer and firewall blockage could be lifted for uploads prior to patients' data submission.

Qualification of PET departments may vary from very detailed with an actual audit (mandatory for PET studies using on-site production of novel radiopharmaceuticals) to only providing the sites with the imaging guidelines and asking them to ascertain whether they are able to comply with the parameters. The IG contain not only detailed acquisition and reconstruction parameters for the research scans of patients in the clinical study, but also guidelines on phantom studies to obtain the necessary approval from the sponsor to use a specific scanner for the study.

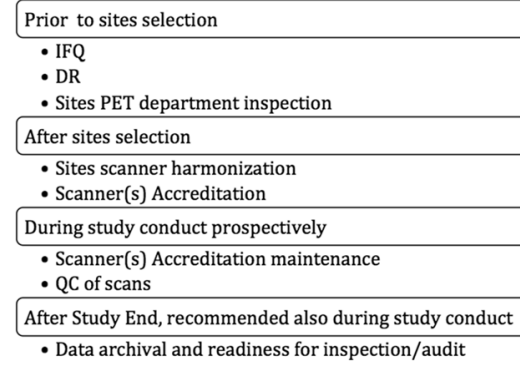

Fig. 1 Steps ensuring data integrity 


\section{After selection of sites}

PET scanner harmonization across different manufacturers has been well studied and documented [15-18]. It is required in order to homogenize scanner performance across all PET makes and models within a multicenter clinical study [19-21]. In addition to uniformity checks, SUV coefficient recovery should be checked either on a regular basis or ad hoc depending on the study needs. Several international organizations have established procedures for this in the multicenter setting across various imaging platforms [22-24]. For well-established IBs such as ${ }^{18}$ F-FDG, accrediting organizations have proposed a yearly check. However, if the study demands rather novel approaches, it may be reasonable to request that sites acquire a phantom scan the day of or prior to imaging a research patient, ensuring that the recovery coefficient is within the acceptable window and that corrections have to be undertaken prior to scanning a research patient, whenever needed.

Scanner accreditation must be obtained prior to the imaging of the first patient in a specific study. In a multicenter PET study, due to variability of makes and models of scanners, it is highly recommended that scanner performance is verified and documented. This is accomplished by ensuring that all PET scanners are accredited by an independent external organization (i.e., European Association of Nuclear Medicine Research Ltd., EARL; Society of Nuclear Medicine and Molecular Imaging Clinical Trial Network, SNMMI CTN; and American College of Radiology Imaging Network, ACRIN) and maintain the accreditation for the period they are participating in the study. Alternatively, regular acquisition of PET phantoms can be implemented. The phantom scans should be processed in an identical manner, using the same methodology and software. After submission to the sponsor for analysis, feedback can be given to the site with specific parameters to be used in the study. The PET scanner accreditation programs [3, 11-13] to date have known differences in assessment, e.g., recovery coefficient analysis for all scanners versus only for some which depend on the study, different software used, and difference in frequency in phantom review. Thus, it may not be appropriate for every study to use a mix of those accrediting agency certifications. For harmonization in scanner performance, it is imperative that the same methodology is used across all sites' scanners utilized in a specific multicenter study.

\section{During the clinical study}

It is highly recommended that a specialized PET imaging monitor visits the PET/ CT department to ascertain that the PET/CT scanner is maintained as recommended by the manufacturer, all daily and periodic image quality control procedures are performed and documented. Scanner performance check should be done not just on a regular basis during the study, but also after every major software update resulting in a scanner software version change and after hardware updates/ changes. During QC it should be checked that the study-qualified scanner is used for the subject's scans in a particular study. Accreditation must be maintained for the duration of the study, or until the last patient's scan is completed, whichever comes first. It is the sponsor's obligation to check and ensure the site maintains its accreditation, but it is the site's responsibility to submit the necessary data to the 
accrediting body and rectify any issues with image quality for the accreditation compliance. It is the site's responsibility to promptly notify the sponsor if the accreditation can no longer be maintained, while there are still study patients scheduled for imaging on the particular scanner.

\section{PET scans QC}

All trials using SUVs as the end points or criteria referencing SUVs, especially referring to longitudinal change such as the recent IWG 2014 lymphoma criteria [14], should implement at the minimum IG and compliance check. While the IG are part of the clinical trial protocol, often are provided segregated from the protocol. The IG detail the role of the PET department, instructions on site's qualification to participate in the study, patient preparation, acquisition, and reconstruction parameters, when the scans have to be acquired and how to submit them.

Prospective detailed QC is recommended for all scans received, and in addition to a check of the DICOM tags for acquisition and reconstruction parameters, study pre-defined normal organ $\mathrm{SUV}_{\text {mean }}$ should be measured (i.e., liver, blood pool, muscle, lungs, and brain). For studies requiring the use of an accredited scanner, check should be done that such a scanner was used. If specific accredited acquisition and reconstruction parameters are required (all follow-up scans should be acquired and reconstructed identically), all scans should be checked against the required parameters. It is important that this is done almost immediately after acquisition and submission. In case of a missing reconstruction, the site would have the possibility to use the raw data still on the scanner and reconstruct and submit as required. Delay in providing QC feedback or site unresponsiveness to reconstruct, could result in loss of data, ultimately affecting the PPP. Raw data is usually overwritten in a matter of days, and it may not be possible for the site to provide further reconstructions.

\section{PET series}

The PET attenuation-corrected (AC), non-attenuation-corrected (NAC), and attenuation correction CT (AC-CT) series should be properly anonymized and submitted to the sponsor and inspected in detail. When reviewing the scan, it should be ascertained that it belongs to the right patient, the specified anatomy in the IG has been scanned, and the images should be assessed for possible artifacts from either the patient or the equipment (i.e., motion, dose infiltration, metal, out of field of view artifacts, truncation artifacts, misregistration, and incorrect attenuation correction). It is very important that this QC is performed by a qualified PET imaging technologist who can recognize the different artifacts and contact the sites suggesting possible remedy prior to a subsequent scan acquisition. The published guidance for industry from the Food and Drug Administration (FDA) specifies that the FDA "anticipate periodic on-site inspection by the trial's imaging-specific monitors to assess the imaging technical compliance of each clinical site or a subset of all the sites" [25]. However, it is not clear if this is done systematically or if and how the FDA checks to ensure this recommendation is implemented. At the minimum the PETAC, NAC, and AC-CT should be submitted for every scan. However, if the 
sites do not submit the NAC series, the impact on the study data would be low, since this series is only used to identify questionable activity concentration during the reviews and to check for patient movement-related artifacts.

\section{Compliance with the visit window schedule}

The scan timing should be confirmed to ensure it is within the specified acceptable window as per the protocol. Any scans outside of pre-specified timing should clearly be identified for the sponsor to ensure proper PPP analysis.

A simple check via a region of interest (ROI) or volume of interest (VOI) with the PET software used for QC should be done to ensure that SUV measurements could be performed and that the data received contains all DICOM tag intact which are necessary and used for SUVs. Validated PET software would produce meaningful values for $\mathrm{SUV}_{\text {max }}, \mathrm{SUV}_{\text {mean }}, \mathrm{SUV}_{\text {peak }}, \mathrm{SUV}_{\text {min }}$, area, and so on. If some of the DICOM tag information are missing, as in a case where the site did not submit the originally reconstructed data but instead submitted post-processed data, the site should be asked to submit the original reconstructions, exported directly from the scanner. It is recommended that sites save this data as it is considered source data and is required to be saved and available upon an audit either from the sponsor or the regulatory authorities. The FDA requires the source data to be saved for at least 2 years after the last drug market authorization; however, local regulations may impose extended time, and the sites should comply with whichever is longer. Some vendor scanners produce DICOM proprietary tags with information required for the SUV measurements (e.g., Private Creator-Philips PET Private Group (7053,0010); Unknown Element-0.002348 (7053,1000)). These tags are often modified or removed by some post-processing software, DICOM-notcompliant software, or during anonymization of the patient data in DICOM. Care should be taken that the original data is saved and available, and only originally reconstructed data is used for SUV measurements.

Often the IG specify clinical data such as fasting time, medication withhold, administration or delay of scan start after a certain treatment is given, blood glucose, and other laboratory tests. This information should be checked during QC against the clinical dossier to ensure verifiability.

The following sections quote specific DICOM tags, and the assumption in this publication is that the readers have access to a DICOM tool and have a basic understanding of the DICOM standards. Table 1 includes detailed DICOM tags with explanations. We suggest using those for a longitudinal check and against the specified parameters in the IG.

Recent treatment interventions or health conditions may affect SUV, e.g., a significant difference in $\mathrm{SUV}_{\max }$-induced by recent chemotherapy [26-28]. When applicable, this information should be available and taken into consideration during QC and in the interpretation of longitudinal PET scans.

Some of the factors noted above, taken by themselves, may not significantly influence SUV measurements, yet a combination of them could result in an erroneous patient category assignment, e.g., eligibility, stratification, and crossover in study arms. Some factors, however, such as the use of point spread function (PSF) within the iterative reconstruction algorithm, could double the change in $\mathrm{SUV}_{\max }$ measurements in pathology [29], while $\mathrm{SUV}_{\text {mean }}$ in normal organ such as the liver will remain almost constant. 


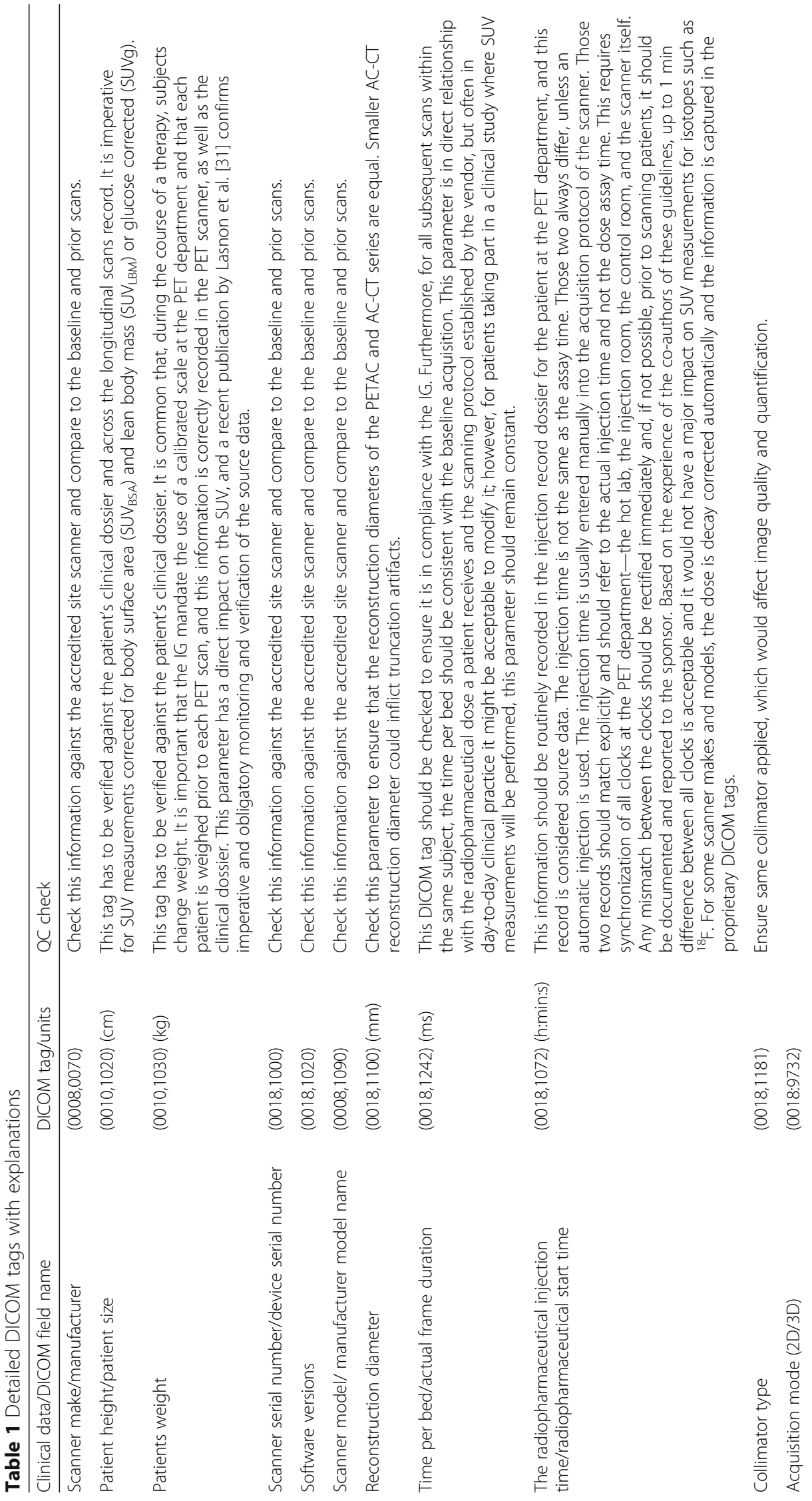




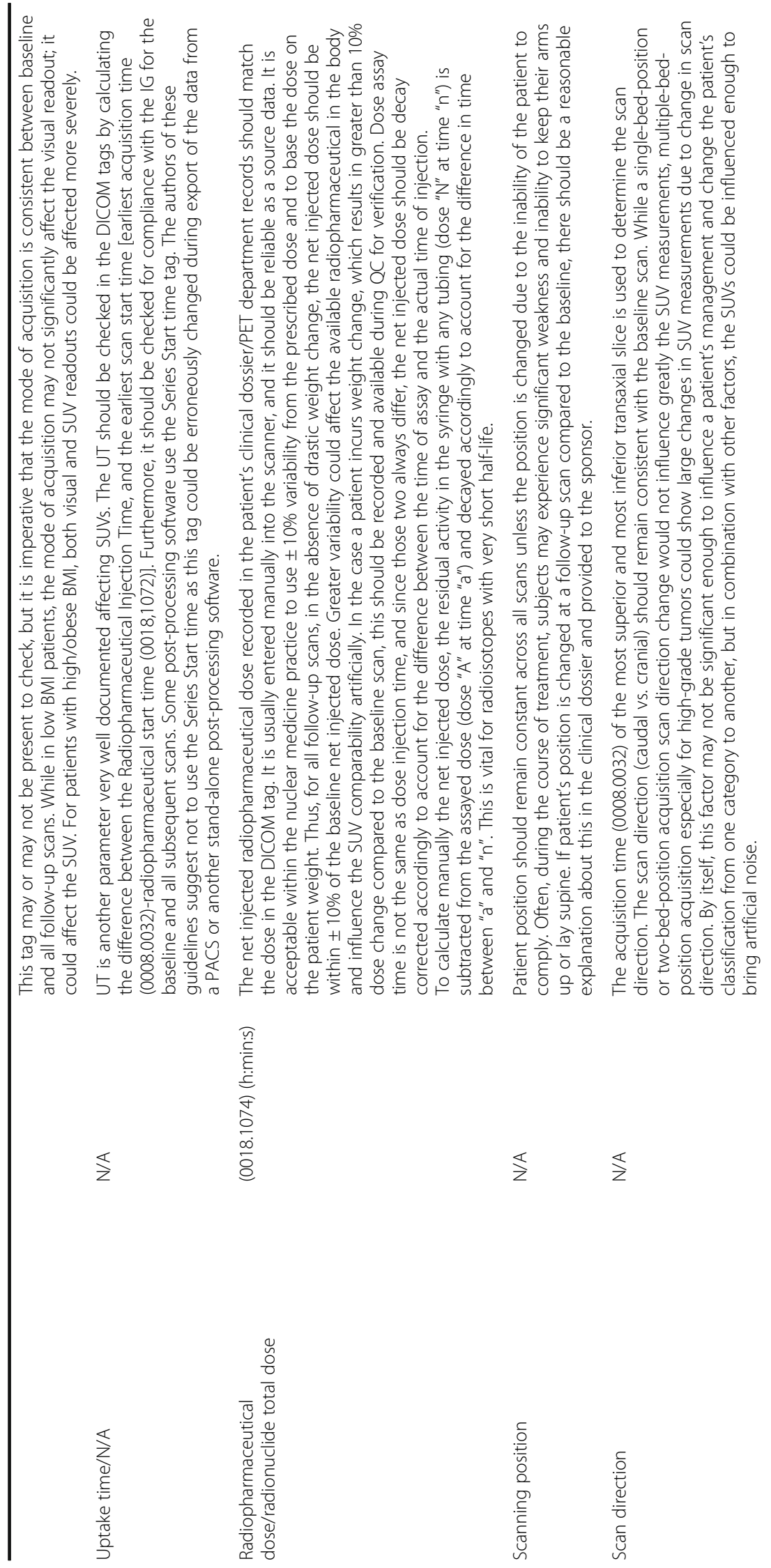




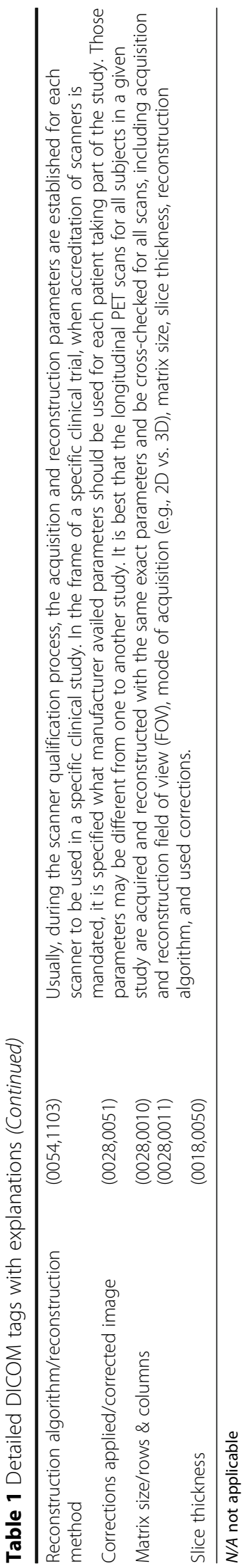


It is important to note that not all systems or vendors provide sufficient information in the DICOM tags about reconstruction type, settings, and/or image processing. Thus, images that have been reconstructed and/or processed differently cannot always be distinguished based on the metadata alone. This only underlines the importance of ensuring that reliable and high-quality data is analyzed.

The use of different reconstruction parameters and the effect on SUVs have been discussed extensively already. Magnitude of effect is variable, and it depends on the different make and model scanners used, as well as the various reconstruction parameters applied. Illustration of such effects is shown here based on a patient scan as follows:

- Table 2 shows the values of SUVs (corrected for body weight (bw), lean body mass (lbm), and body surface area (bsa)) of a single lesion, large enough to be selected as a target lesion, and a whole body total glycolytic activity of total body tumor burden for two different reconstructions

- Table 3 shows the values of SUV (corrected for bw, lbm, and bsa) of normal tissue (blood pool, liver, muscle, lung) using the site standard of care PSF reconstruction and EARL reconstruction

- Figure 2a (single lesion) and b (whole body tumor burden maximum intensity projection) shows the images. In Fig. 2b, the whole body was first segmented based on the AC-CT to include skin to skin anatomy in 3D. The VOI from this section is then copied to the two PETAC reconstructions and sub-segmented to include only SUV values above 2.5, and normal tissue uptake was subtracted (i.e., brain and kidney activity).

Informed consent was obtained from all individual participants whose data was used in this article.

SUV measurements were performed using MIM Vista software (MIM Software Inc., Cleveland, OH USA). In this case, the data presented in the DICOM tags is identical as to acquisition and reconstruction parameters. However, as shown via the SUV measurements, it is clear that the reconstructions are different.

Radiopharmaceutical administration should be performed only via saline-tested and well patent IV access. Extravasation is rare, however, in the case of observed extravasation affecting the study quality, and ultimately rendering the quantification incorrect, scan repeat might be necessary when possible.

Changes in SUV used for patient management, or for clinical studies end points, should be based on true, high-quality data, verified to ensure that technical factors have been excluded as a source of error. Without detailed QC of the parameters described in these guidelines, it is possible that treatment allocation is made merely based on factors, which could mislead one way or the other, leading either in change in treatment strategy or not. In both instances, the results are devastating to patients. As a whole, increased noise in the data of a clinical study and questionable quality of the applied methods, undermine the validity of study conclusions and may lead to a clinical study failure or to an ultimate positive result, which contains invalid source data. Rarely, if ever, the regulatory authorities check to ensure that for surrogate end points the data presented is, in fact, free of 


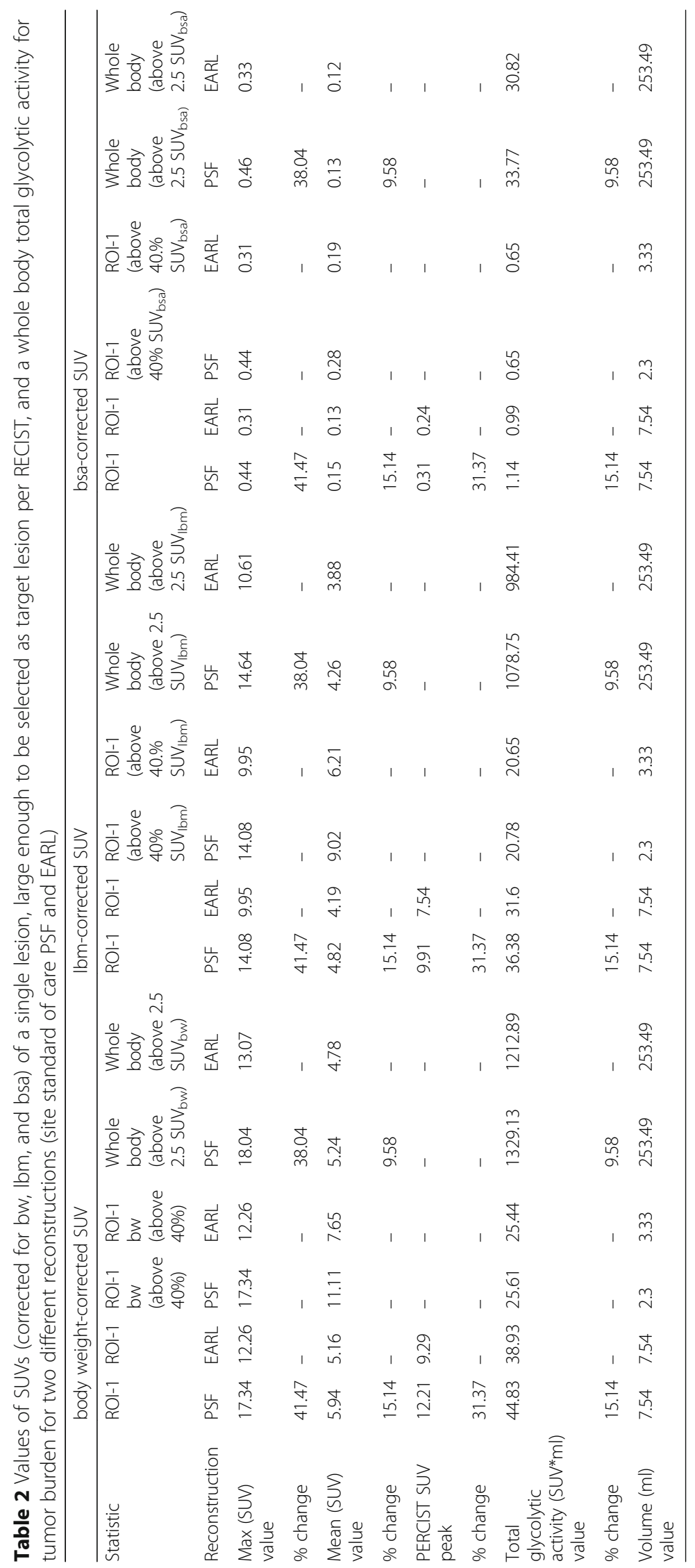




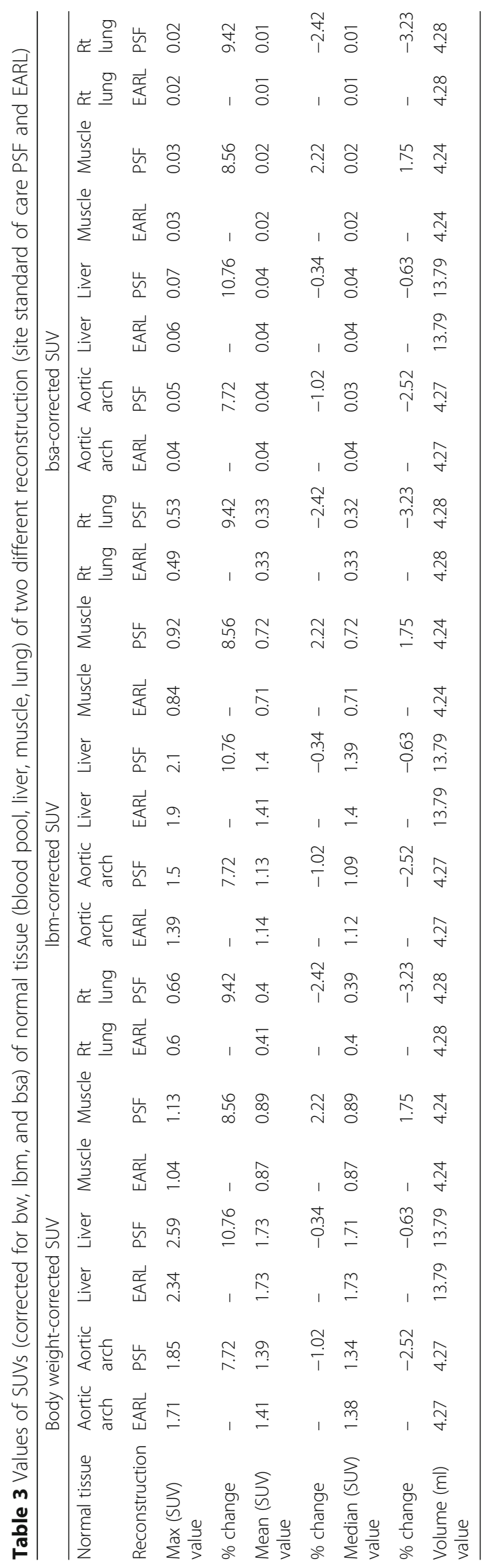




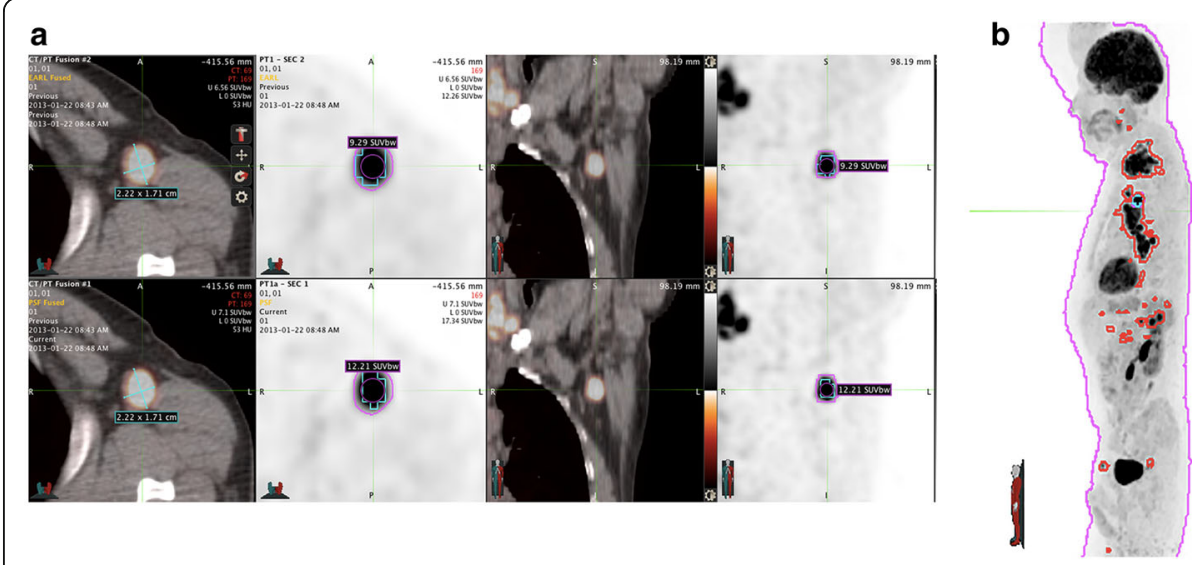

Fig. 2 a Single lesion volume of interests (1) for entire lesion with (2) RECIST measurement and (3) 40\% SUV threshold. $\mathbf{b}$ Whole body tumor burden maximum intensity projection-segmented based on the AC$C T$, contour was then copied to the two PETAC reconstructions and sub-segmented to include only SUV values above 2.5 and normal tissue uptake was subtracted (i.e., brain and kidney)

technical artifacts. While the latest FDA guidelines from March 2015 [30] include more stringent points on quality, it is not known or clear if any of the additional suggestions made will be implemented, checked, and complied with in the future.

\section{After study completion}

After study completion, it must be ensured that the data are properly archived and ready for inspection by either the regulatory agencies or the sponsor. Any changes to the digital data (i.e., DICOM tag update) must be done in an audit trail fashion, ensuring the ability to track the changes per the specified regulations.

Implementing the steps suggested in Fig. 1 as detailed above, prior and after site selection, during and at end of a study, would ensure the integrity of the data, the scientific value, and boost trust in results and would ultimately help patients in the fight of disease. Verifiable quality data, even in a small number of patients, is the future of IB use as surrogate end points in clinical trials.

\section{Summary}

\section{Concluding remarks}

While daily clinical standard of care variations is a fact, bringing noise in the day to day practice, validation of SUVs as IB aims to set out an international standard to be relied on; thus, the quality of the data for the IB must be held to the highest standards. Assuring the quality of imaging data as proposed in these guidelines aim to safeguard the validity of imaging data and study conclusions, which is relevant for scientific journals, the regulatory authorities, and the sponsors. Implementation of rigorous QC procedures ensures that basic data affecting SUVs are verified prior to readouts. In the absence of $\mathrm{QC}$, readouts could be merely a reflection of technical factors, and conclusions about the validity of PET as an IB may be inappropriate. 
Availability of data and materials

The datasets of the images supporting the conclusions of this article may be made available upon request.

\section{Authors' contributions}

$I H, R B$, and WJGO made substantial contributions to conception and design, or acquisition of data, or analysis and interpretation of data. $\mathrm{H}, \mathrm{RB}, \mathrm{PG}, \mathrm{LKS}, \mathrm{YL}, \mathrm{SS}, \mathrm{OSH}$, and WJGO have been involved in drafting the manuscript or revising it critically for important intellectual content. $I H, R B, P G, L K S, Y L, S S, O S H$, and WJGO gave final approval of the version to be published. Each author should have participated sufficiently in the work to take public responsibility for appropriate portions of the content. IH, RB, PG, LKS, YL, SS, OSH, and WJGO agreed to be accountable for all aspects of the work in ensuring that questions related to the accuracy or integrity of any part of the work are appropriately investigated and resolved.

\section{Ethics approval and consent to participate}

All procedures performed in studies involving human participants were in accordance with the ethical standards of the institutional and/or national research committee and with the 1964 Helsinki declaration and its later amendments or comparable ethical standards. However, this study includes a retrospective analysis, and for this type of study, formal consent is not required.

\section{Consent for publication}

Informed consent was obtained from all individual participants included in the study.

\section{Competing interests}

The authors declare that they have no competing interests.

\section{Author details}

${ }^{1}$ Department of Nuclear Medicine, Radboud University Medical Centre, Geert Grooteplein-Zuid 10, 6525 GA Nijmegen, The Netherlands. ${ }^{2}$ University Medical Center Groningen, University of Groningen, Groningen, The Netherlands. ${ }^{3}$ The Institute of Cancer Research, London, UK. ${ }^{4}$ European Organization for Research and Treatment of Cancer, Imaging Group, Brussels, Belgium. ${ }^{5}$ GSK, Experimental Medicine Imaging, Upper Providence, PA, USA. ${ }^{6}$ Division of Cancer

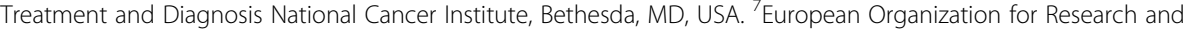
Treatment of Cancer, Headquarters, Brussels, Belgium. ${ }^{8}$ Molecular Imaging Center Antwerp (MICA), Faculty of Medicine and Health Sciences, University of Antwerp, Wilrijk, Belgium. ${ }^{9}$ Department of Radiology \& Nuclear Medicine, VU University Medical Centre, Amsterdam, NL, The Netherlands.

Received: 15 February 2017 Accepted: 6 September 2017

Published online: 18 September 2017

\section{References}

1. Boellaard R. Standards for PET image acquisition and quantitative data analysis. J Nucl Med May 2009 vol. 50 no. Suppl 1 11S-20S.

2. Doot RK, Pierce LA, Byrd D, Elston B, Allberg KC, Kinahan PE. Biases in multicenter longitudinal PET standardized uptake value measurements. Transl Oncol. 2014 Feb;7(1):48-54. Published online 2014 Feb 1

3. Vriens D, Visser EP, de Geus-Oei LF, Oyen WJ. Methodological considerations in quantification of oncological FDG PET studies. Eur J Nucl Med Mol Imaging. 2010 Jul;37(7):1408-25. Published online 2009 Nov 20

4. Fahey FH, Kinahan PE, Doot RK, Kocak M, Thurston H, Poussaint TY. Variability in PET quantitation within a multicenter consortium. Med Phys. 2010 Jul;37(7):3660-6. Published online 2010 Jun 21

5. Shankar LK, Hoffman JM, Bacharach S, Graham MM, Karp J, Lammertsma AA, Larson S, Mankoff DA, Siegel BA, Van den Abbeele A, Yap J, Sullivan D. Consensus recommendations for the use of 18F-FDG PET as an indicator of therapeutic response in patients in National Cancer Institute Trials. J Nucl Med. 2006 Jun;47(6):1059-66.

6. Hristova I, Boellaard R, Vogel W, Mottaghy F, Marreaud S, Collette S, Schöffski P, Sanfilippo R, Dewji R, van der Graaf W, Oyen WJ. Retrospective quality control review of FDG scans in the imaging sub-study of PALETTE EORTC 62072NEG110727: a randomized, double-blind, placebo-controlled phase III trial. Eur J Nucl Med Mol Imaging. 2015 May;42(6):848-57. doi: 10.1007/s00259-015-3002-0. Epub 2015 Feb 25

7. Geus-Oei LF, van der Heijden HF, Corstens FH, Oyen WJ. Predictive and prognostic value of FDG-PET in nonsmallcell lung cancer: a systematic review. Cancer. 2007;110(8):1654-64.

8. Quantitative Imaging Biomarkers Alliance. Quantitative FDG-PET Technical Committee. UPICT oncology FDG-PET CT protocol. http://qibawiki.rsna.org/index.php?title=FDG-PET_tech_ctte Accessed 10 Dec 2015

9. Wahl RL, Jacene $H$, Kasamon $Y$, Lodge MA. From RECIST to PERCIST: evolving considerations for PET response criteria in solid tumors. J Nucl Med. 2009;50(Suppl 1):122S-50.

10. Young H, Baum R, Cremerius U, et al. Measurement of clinical and subclinical tumour response using [18F]fluorodeoxyglucose and positron emission tomography: review and 1999 EORTC recommendations. European Organization for Research and Treatment of Cancer (EORTC) PET Study Group. Eur J Cancer. 1999;35(13):1773-82.

11. Itti E, Meignan M, Berriolo-Riedinger A, et al. An international confirmatory study of the prognostic value of early PET/CT in diffuse large B-cell lymphoma: comparison between Deauville criteria and DeltaSUVmax. Eur J Nucl Med Mol Imaging. 2013;40(9):1312-20.

12. Chung $\mathrm{HH}$, Kwon HW, Kang KW, et al. Prognostic value of preoperative metabolic tumor volume and total lesion glycolysis in patients with epithelial ovarian cancer. Ann Surg Oncol. 2012;19(6):1966-72.

13. Zhang $\mathrm{H}$, Wroblewski $\mathrm{K}$, Liao $\mathrm{S}$, et al. Prognostic value of metabolic tumor burden from (18)F-FDG PET in surgical patients with non-small-cell lung cancer. Acad Radiol. 2013;20(1):32-40. 
14. Barrington SF, Mikhaeel NG, Kostakoglu L, et al. Role of imaging in the staging and response assessment of lymphoma: consensus of the international conference on malignant lymphomas imaging working group. J Clin Oncol. 2014

15. EANM Research Ltd. New EANM FDG PET/CT accreditation specifications for SUV recovery coefficients. Vienna: EANM Research Ltd; 2011. http://earl.eanm.org/cms/website.php?id=/en/projects/fdg_pet_ct_accreditation/ accreditation_specifications.htm.

16. Sunderland JJ, Christian PE. Quantitative PET/CT scanner performance characterization based upon the Society of Nuclear Medicine and Molecular Imaging Clinical Trials Network oncology clinical simulator phantom. J Nucl Med. 2015;56(1):145-52.

17. Makris NE, Huisman MC, Kinahan PE, Lammertsma AA, Boellaard R. Evaluation of strategies towards harmonization of FDG PET/CT studies in multicentre trials: comparison of scanner validation phantoms and data analysis procedures. Eur J Nucl Med Mol Imaging. 2013;40(10):1507-15.

18. Radiological Society of North America. FDG-PET/CT Technical Committee. FDG-PET/CT as an imaging biomarker measuring response to cancer therapy. Quantitative Imaging Biomarkers Alliance. Version 1.05. Publicly reviewed version. QIBA, 11 December 2013. http://www.rsna.org/uploadedfiles/rsna/content/science_and_education/qiba/ qiba_fdgpet_profile_v105_publicly_reviewed_version_final_11dec2013.pdf.

19. Beyer T, Czernin J, Freudenberg LS. Variations in clinical PET/CT operations: results of an international survey of active PET/CT users. J Nucl Med. 2011;52(2):303-10. doi: 10.2967/jnumed.110.079624

20. Graham MM, Badawi RD, Wahl RL. Variations in PET/CT methodology for oncologic imaging at U.S. academic medical centers: an imaging response assessment team survey. J Nucl Med. 2011;52(2):311-7. doi: 10.2967/ jnumed.109.074104

21. Rausch I, Bergmann H, Geist B, Schaffarich M, Hirtl A, Hacker M, et al. Variation of system performance, quality control standards and adherence to international FDG-PET/CT imaging guidelines. A national survey of PET/CT operations in Austria. Nuklearmedizin. 2014;53(6):242-8. doi: 10.3413/Nukmed-0665-14-05

22. http://www.ctndatabase.org Accessed 10 Dec 2015

23. http://www.acr.org/quality-safety/accreditation Accessed 10 Dec 2015.

24. http://earl.eanm.org/cms/website.php?id=/en/projects/fdg_pet_ct_accreditation.htm Accessed 10 Dec 2015.

25. http://www.fda.gov/downloads/Drugs/GuidanceComplianceRegulatorylnformation/Guidances/UCM268555.pdf Accessed 10 Dec 2015.

26. Cheebsumon P, Velasquez LM, Hoekstra CJ, Hayes W, Kloet RW, Hoetjes NJ, Smit EF, Hoekstra OS, Lammertsma AA, Boellaard R. Measuring response to therapy using FDG PET: semi-quantitative and full kinetic analysis. Eur J Nucl Med Mol Imaging. 2011;38(5):832-42. doi:10.1007/s00259-010-1705-9. Epub 2011 Jan 6.

27. Doot RK, Dunnwald LK, Schubert EK, Muzi M, Peterson LM, Kinahan PE, et al. Dynamic and static approaches to quantifying 18F-FDG uptake for measuring cancer response to therapy, including the effect of granulocyte CSF. J Nucl Med. 2007;48:920-5. doi: 10.2967/jnumed.106.037382

28. Freedman NM, Sundaram SK, Kurdziel K, Carrasquillo JA, Whatley M, Carson JM, et al. Comparison of SUV and Patlak slope for monitoring of cancer therapy using serial PET scans. Eur J Nucl Med Mol Imaging. 2003;30:46-53. doi: 10.1007/s00259-002-0981-4

29. Kuhnert G, Boellaard R, Sterzer S, Kahraman D, Scheffler M, Wolf J, Dietlein M, Drzezga A, Kobe C. Impact of PET/ $\mathrm{CT}$ image reconstruction methods and liver uptake normalization strategies on quantitative image analysis. Eur J Nucl Med Mol Imaging. 2016 Feb;43(2):249-58. doi: 10.1007/s00259-015-3165-8. Epub 2015 Aug 18

30. http://www.fda.gov/downloads/drugs/guidancecomplianceregulatoryinformation/guidances/ucm268555.pdf Accessed 10 Dec 2015.

31. Lasnon C, Houdu B, Kammerer E, Salomon T, Devreese J, Lebasnier A, Aide N. Patient's weight: a neglected cause of variability in SUV measurements? A survey from an EARL accredited PET centre in 513 patients. Eur J Nucl Med Mol Imaging. 2015 Oct; 17 [Epub ahead of print]

Submit your manuscript to a SpringerOpen ${ }^{\odot}$ journal and benefit from:

- Convenient online submission

- Rigorous peer review

Open access: articles freely available online

High visibility within the field

- Retaining the copyright to your article

Submit your next manuscript at $\gg$ springeropen.com 